\title{
Sepsisquelle Abdomen - zwischen interventioneller, chirurgischer und intensivmedizinischer Therapie
}

\author{
Elke Muhl ${ }^{\mathrm{a}}$ Klaus Fellermann ${ }^{\mathrm{b}}$ \\ ${ }^{a}$ Chirurgische Intensivstation, Klinik für Chirurgie, \\ ${ }^{\mathrm{b}}$ Bereich Gastroenterologie, Medizinische Klinik I, Universitätsklinikum Schleswig-Holstein, Campus Lübeck, Deutschland
}

Die kausale Behandlung der Sepsis bedeutet die Kontrolle des septischen Fokus; sie muss zeitnah erfolgen und ist die Voraussetzung für den Erfolg der Sepsistherapie [1]. Die Therapie der Sepsisquelle im Abdomen ist dabei schon längst nicht mehr ein rein chirurgisches Handlungsfeld. Laparoskopische Verfahren haben auch in der Chirurgie des septischen Fokus im Abdomen Einzug gehalten, und Viszeralchirurgen betätigen sich endoskopisch, z.B. mit der transanalen endoskopischen Mikrochirurgie (TEM).

Der Gastroenterologe ist in die Behandlung von Nahtinsuffizienzen am Gastrointestinaltrakt - nach offener oder laparoskopischer Chirurgie - eingebunden. In ausgewählten Fällen sind Clipping, Stent oder endoskopisch angelegte Vakuumschwammtherapie möglich und sinnvoll.

Septische Komplikationen sind die häufigsten Komplikationen nach viszeralchirurgischen Eingriffen. Gleichzeitig nimmt der Anteil der laparoskopischen Eingriffe in der Viszeralchirurgie zu. Daher liegt es nahe, auch die Komplikationen der Nahtinsuffizienz einer minimal invasiven Therapie zuzuführen - sei es laparoskopisch, sei es endoskopisch -, um dem Patienten die Vorteile des initial laparoskopischen Vorgehens zu erhalten. Das Kapitel von Werner und Sauer [2] beleuchtet die Differentialindikationen dieser Verfahren und ihre Erfolgsaussichten bei Nahtinsuffizienzen gastrointestinaler Anastomosen.

Vakuumschwammtherapie und Stent werden nicht selten in Kombination mit radiologisch-interventionell gelegten Drainagen angewandt, über die in der Umgebung der Nahtinsuffizienz gelegene Abszesse drainiert werden können. Die diagnostische Punktion intraabdomineller Flüssigkeitsverhalte und die therapeutische Drainage ist bei vielen Lokalisationen im Abdomen CT-gesteuert möglich und stößt an ihre Grenzen, wenn die Sekrete nicht rein liquide sind (z.B. bei infizierten Pankreasnekrosen) oder wenn im Zugangsweg
Strukturen liegen, die nicht verletzt werden dürfen, wie Wiggermann und Hunold [3] in ihrem Beitrag darstellen.

Infizierte Pankreasnekosen sind vielfach einer endoskopischen Drainage und Lavage zugänglich - ein Verfahren, das einer subtilen Indikationsstellung im Einzelfall bedarf und das - wie auch die laparoskopischen Verfahren in der Viszeralchirurgie - von der Erfahrung des Endoskopikers abhängig ist, wie Fellermann [4] schildert. Ist das Verfahren anwendbar, erspart es dem Patienten große und potenziell komplikationsträchtige offen-operative Eingriffe oder kann im Rahmen einer schrittweisen Herangehensweise der Einstieg sein.

Die laparoskopische Chirurgie hat auch beim Notfalleingriff mit abdomineller Sepsis ihren Platz: Keck, Wellner und Karcz [5] heben in ihrer Arbeit die Vorteile des laparoskopischen Vorgehens hervor, d.h. unter anderem geringerer Schmerzmittelbedarf, kürzere Verweildauer und verminderte Wundinfektionsraten. Die Erfahrung des Operateurs spielt hierbei eine nicht zu unterschätzende Rolle für das Gelingen des laparoskopischen Eingriffs - mit einer Operationsdauer sowie mit Komplikationsraten, die dem offenen Verfahren gleichkommen. Die minimal invasive retroperitoneoskopische Nekrosektomie infizierter Pankreasnekrosen ist - bei richtiger Indikationsstellung, bei Kombination mit anderen nichtinvasiven Verfahren und bei entsprechender Expertise des Operateurs - oft erfolgreich und stellt eine elegante Alternative zum offenen transabdominellen Verfahren dar. Bei Gelingen kann durch das retroperitoneoskopische Verfahren eine Verschleppung der Infektion in das Abdomen vermieden werden.

Die möglichst frühzeitige antiinfektive Chemotherapie und die Intensivtherapie mit hämodynamischer Stabilisierung durch Volumentherapie und Behandlung mit Vasopressoren und Inotropika, aber auch die Behandlung sämtlicher assozi-

\section{KARGER \\ Fax +497614520714 \\ Information@Karger.com}

www.karger.com (c) 2013 S. Karger GmbH, Freiburg

$1662-6664 / 13 / 0291-0005 \$ 38.00 / 0$

Accessible online at:

www.karger.com/vim
Prof. Dr. Elke Muhl

Chirurgische Intensivstation, Klinik für Chirurgie

Universitätsklinikum Schleswig-Holstein, Campus Lübeck

Ratzeburger Allee 160, 23538 Lübeck, Deutschland

elke.muhl@uk-sh.de 
ierter Organdysfunktionen sind ein integrativer Bestandteil der Sepsistherapie in der Intensivmedizin. Einem dieser Aspekte, der Volumentherapie, ist das Kapitel von Muhl [6] gewidmet. In der elektiven Viszeralchirurgie haben prospektive Studien Vorteile für ein restriktives Volumenmanagement gezeigt, während bei schwerer Sepsis gerade initial ausgeprägt positive Flüssigkeitsbilanzen erforderlich werden, um Zielparameter der Volumentherapie zu erreichen.
Der Patient mit abdomineller Sepsis auf der Intensivstation sollte, wann immer möglich, enteral ernährt werden. Auch bei funktionellen Passagestörungen ist dies in aller Regel zumindest partiell möglich, wie Kuppinger und Hartl erläutern [7]. Bei den Intensivpatienten, die mit hohen Dosen an Opiaten behandelt werden müssen, ist auch an die Therapie der Darmatonie mit einem $\mu$-Rezeptorantagonisten (Methylnaltrexoniumbromid) zu denken.

\section{Literatur}

1 Reinhart K, Brunkhorst FM, Bone HG, et al.: Prevention, diagnosis, therapy and follow-up care of sepsis: 1st revision of S2k guidelines of the German Sepsis Society (Deutsche Sepsis Gesellschaft e.V. (DSG)) and the German Interdisciplinary Association of Intensive Care and Emergency Medicine (Deutsche Interdisziplinäre Vereinigung für Intensiv- und Notfallmedizin (DIVI)). Ger Med Sci 2010;8:Doc14
2 Werner J, Sauer P: Nahtinsuffizienz intestinaler Anastomosen: Endoskopische und laparoskopische Therapieoptionen. Viszeralmedizin 2013;29:7-13.

3 Wiggermann A, Hunold P: Radiologisch-interventionelle Drainage bei abdomineller Sepsis. Viszeralmedizin 2013;29:14-20.

4 Fellermann K: Endoskopie als Baustein der interventionellen Therapie infizierter Pankreasnekrosen. Viszeralmedizin 2013;29:21-26.
5 Keck T, Wellner U, Tittelbach-Helmrich D, Bausch D, Karcz K: Grenzen des laparoskopischen Operierens bei abdomineller Sepsis. Viszeralmedizin 2013;29:28-33.

6 Muhl E: Volumentherapie in der Sepsis: Wann ist das Limit erreicht? Viszeralmedizin 2013;29:34-40.

7 Kuppinger D, Hartl WH: Spezielle Aspekte der Ernährung bei Patienten mit abdomineller Sepsis. Viszeralmedizin 2013;29:41-45. 